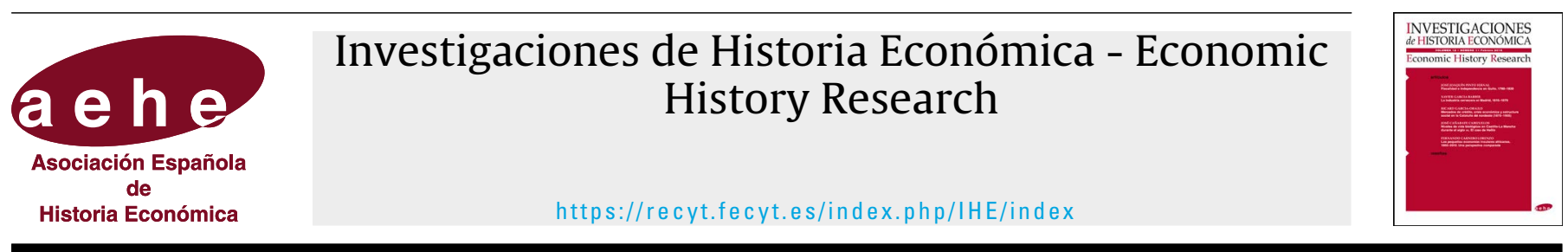

Artículo

\title{
Hacia un marco analítico de las consecuencias demográficas y económicas de las epidemias
}

\section{Vicente Pérez Moreda* \\ Universidad Complutense de Madrid}

\section{INFORMACIÓN DEL ARTÍCULO}

\section{Historia del artículo:}

Recibido: 1 julio 2020

Aceptado: 30 septiembre 2020

On-line: 1 diciembre 2020

\section{Códigos JEL:}

I19

J19

N30

Q54

Palabras clave:

Epidemias

Economía de la salud

Historia económica

España

\begin{abstract}
R E S U M E N
Con motivo de la actual pandemia de COVID-19 se ha puesto a disposición de los investigadores una enorme cantidad de fuentes clásicas y recientes sobre las grandes pandemias y su impacto demográfico, social y económico. Con la ayuda parcial de ese material se presentan aquí unas reflexiones que intentan configurar un marco conceptual y analítico de las conexiones entre las manifestaciones históricas de la morbilidad y mortalidad epidémicas y sus efectos demográficos y económicos. Aunque las consecuencias concretas del shock epidémico deberán verse confirmadas por el estudio de la experiencia histórica en cada caso, una exposición sistemática como la que aquí se ofrece podría servir como punto de partida de análisis posteriores que valoren debidamente la importancia de los cambios introducidos en la población y en la economía por las epidemias y pandemias del pasado.
\end{abstract}

@ 2020 Asociación Española de Historia Económica

\section{In search of an analytical framework for the study of demographic and economic consequences of epidemics}

\author{
A B S T R A C T
}

In light of the current Covid-19 pandemic a considerable amount of bibliographical and documentary resources have been made available for the study of historical communicable diseases and their demographic, social and economic implications. Based to some extent on this literature, we offer a conceptual and analytical framework of the link between those epidemic crises and their demographic, social and economic impact on past populations. Even though the consequences of each specific epidemic shock must be confirmed by the corresponding historical data, their systematic explanation can represent a useful starting point for future assessments of the demographic and economic changes induced by historical epidemics and pandemics.

@ 2020 Asociación Española de Historia Económica

Epidem

Health economics

Economic history

Spain

We need an ethnography as well as an ecology to explain the network of interactions underlying the appearance, diminution or recrudescence of particular infectious ills [...]. Without a history and political economy we can have neither a meaningful ethnology nor a meaningful ecology. And certainly we cannot have an effective epidemiology.

\footnotetext{
* Autor de correspondencia. Correo electrónico: vperezmo@ucm.es 


\section{Introducción}

La irrupción, inesperada para la mayoría de los habitantes del planeta, de la actual pandemia de SARS-Cov-2, la rápida sucesión de cifras cada vez más abultadas de morbilidad y mortalidad y la inmediata aparición de profundas consecuencias sociales y económicas, con indicadores de recesión que no se conocían desde hace muchas décadas, han despertado el interés de los historiadores o la necesidad de acudir a la historia en búsqueda de referencias útiles que sirvan para situar en sus justos términos la magnitud y gravedad de la crisis actual y que, incluso, puedan guiar las medidas que han de recomendar y aplicar los responsables técnicos y políticos.

Para ello se han movilizado recursos -principalmente el acceso libre a las fuentes de datos y a la bibliografía sobre los temas relacionados con la enfermedad y sus diversas implicaciones-, con el propósito de poner en contacto a los investigadores de distintas disciplinas. También los historiadores de diversas especialidades han sido convocados al análisis y discusión de los sucesos epidémicos del pasado, cuyas características, circunstancias y consecuencias ofrecen una clara relación con las que presenta la pandemia actual. Así, diversas asociaciones e instituciones académicas y otras importantes plataformas de discusión científica, más allá de nuestras fronteras, han reeditado trabajos "clásicos" sobre las grandes enfermedades transmisibles del pasado o han divulgado textos originales y proyectos sobre la actual pandemia de COVID-19, que también suelen incluir, de forma parcial al menos, material relativo a la historia económica o demográfica de las epidemias históricas ${ }^{1}$. En Italia, donde se centraliza la publicación periódica de breves trabajos demográficos -Neodemos-, se ha publicado una interesante selección de los relativos a la COVID-19 y a otras epidemias históricas, con especial referencia al caso italiano (Livi Bacci, 2020); y en Brasil aparece otra publicación de este tipo, aún más amplia y pluridisciplinar (Cássia da Silva et al., 2020). En España también han surgido iniciativas similares por parte de la Asociación Española de Geografía (2020), la Fundación de Ciencias de la Salud (2020) y el CSIC (2020a, 2020b). La Asociación de Demografía Histórica ha publicado una bibliografía de artículos aparecidos desde 1988 en la Revista de Demografía Histórica sobre epidemias y crisis de mortalidad; la Sociedad Española de Historia de la Medicina ha reunido en un instructivo volumen digital Cuarenta historias para una cuarentena (Campos et al., 2020), y varios blogs mantienen abiertas secciones sobre la pandemia y la historia de las

\footnotetext{
${ }^{1}$ Tres series de referencias bibliográficas sobre epidemias y crisis demográficas del pasado aparecieron en The Long Run, el blog de la Economic History Society, e incluían artículos de la Economic History Review, el Journal of Economic History, Explorations in Economic History o la European Review of Economic History (Economic History Society, 2020a, 2020b, 2020c). El Institut National d'Études Démographiques (INED) francés difundió la serie "Pandémies et histoire. Crise sanitaire et confinement: l'apport de la démographie et des sciences de la population", con artículos de Population y otras revistas internacionales (INED, 2020). La Société de Démographie Historique (SDH) ha hecho otro tanto con otra relación de trabajos aparecidos en los Annales de Démographie Historique (SDH/ADH, 2020). La American Anthropologist Association (2020) recopila una muestra de artículos sobre "Disease and Pandemics» aparecidos entre 1985 y 2017, y la newsletter Above the Fold (Project Syndicate) ofrece desde el pasado mes de abril entregas semanales de trabajos originales, firmados muchos de ellos por famosos especialistas, sobre historia económica, política económica, epidemiología y políticas sanitarias.
}

epidemias ${ }^{2}$. En este contexto se encuadran las iniciativas de la Asociación Española de Historia Económica (AEHE) de publicar en su web una selección de trabajos clásicos y nuevas contribuciones sobre el tema (AEHE, 2020), y la de dedicar este número especial de Investigaciones de Historia Económica-Economic History Research a la historia económica y social de las pandemias.

Son muchísimas, pues, inabordables para el investigador individual, las fuentes disponibles para establecer un mínimo estado de la cuestión que intente resumir lo esencial de los estudios sobre la mortalidad epidémica en la historia. Por ello, las páginas que siguen se limitarán a presentar el marco teórico de las principales consecuencias demográficas y económicas del impacto epidémico; lo que podría servir, con la ayuda de adicionales aportaciones procedentes de la epidemiología y la economía de la salud, para elaborar futuros análisis de las epidemias y pandemias del pasado, y de sus implicaciones económicas en concreto, con alguna alusión eventual a las manifestaciones sociales e implicaciones políticas relacionadas con ellas. La previsible continuidad de la actual pandemia de COVID-19 seguirá impulsando su estudio con urgencia y nos dará ocasión para reflexionar sobre sus similitudes y contrastes con las catástrofes de naturaleza próxima que la han precedido en un pasado más o menos reciente.

\section{Reacciones demográficas y consecuencias económicas de la epidemia}

La primera manifestación de una irrupción epidémica es, por definición, la del alza de la mortalidad y de la morbilidad específica. A partir de ahí, las reacciones demográficas se aprecian comúnmente, más pronto o más tarde, en los restantes componentes del movimiento natural (nupcialidad y fecundidad), en el movimiento migratorio y en la estructura por edades y sexo de la población.

Guardando por lo general relación con la gravedad de la crisis epidémica -esto es, con la magnitud del exceso de morbilidad y mortalidad que origine-, se advierte un retroceso temporal de la nupcialidad y de las concepciones, con el resultado de una caída de la fecundidad varios meses después, visible sobre todo en las cifras anuales de nacimientos del año civil posterior. Ambos movimientos a la baja de los mecanismos de la reproducción se explican por la conducta prudencial de sus protagonistas, por el clima de dramatismo de las circunstancias del momento, incompatibles con los festejos $-\mathrm{y}$ los gastos- nupciales, y también, en el caso del cese o retroceso de las concepciones, por la influencia de las doctrinas religiosas y médicas, que aconsejaban la restricción de las relaciones sexuales durante la enfermedad (Bennassar, 1969, p. 51; Biraben, 1975)3. En el caso de la caída de la nupcialidad, se debe tener en cuenta una motivación añadida puramente demográfica, que es la distorsión del mercado matrimonial y la misma imposibilidad de celebrar enlaces ya consensuados con

\footnotetext{
${ }^{2}$ Conversación sobre la Historia (https://conversacionsobrehistoria.info/) o Nada es gratis (https://nadaesgratis.es/).

${ }^{3}$ Los tratadistas españoles de la peste del siglo XVI -P. Ciruelo, Andrés Laguna, Luis de Mercado-, citando alguno de ellos los consejos de san Pablo, recomiendan a los casados que «duerman separados» y desaconsejan «el acceso desordenado a las Damas... mayormente quando reyna esta mala influentia» (Carreras Panchón, 1976, pp. 104, 118, 150).
} 
anterioridad a causa de la mortalidad de alguno de los contrayentes o de ambos. También el descenso de las concepciones puede atribuirse parcialmente a las reacciones demográficas desatadas por la misma epidemia: un menor número de bodas y la sobremortalidad de adultos -entre ellos, de mujeres gestantes- explicarían en parte la caída posterior de los nacimientos de primer orden y de los nacimientos en general, respectivamente.

No todos los autores han aceptado que estas consecuencias sobre la nupcialidad y la fecundidad, que eran temporales y revertían pronto en forma de rebotes compensatorios una vez superada la crisis, sean aplicables a los casos de sobremortalidad de naturaleza epidémica. Los partidarios de la teoría de las crisis de subsistencias las relacionaban solamente con estas últimas, derivadas de grandes carestías, hambrunas o graves problemas alimentarios en general, descartando la posibilidad de que en las crisis epidémicas se registraran variaciones notables en la fecundidad o la nupcialidad (Goubert, 1965; Meuvret, 1965a, 1965b)4. Posteriormente, otros autores rechazaron esta interpretación restrictiva, pues de hecho tales consecuencias se manifestaban en todas las mortandades del pasado con independencia de su origen o naturaleza; por lo demás, en la mayor parte de los casos no se trataba de crisis clasificables exclusivamente como "de subsistencias» o como «epidémicas», sino que más bien eran de naturaleza mixta (Appleby, 1973 y 1977; Biraben, 1975; Pérez Moreda, 1980, pp. 94-98). Sobre la duración efímera de estas consecuencias y la reacción compensatoria del crecimiento natural mediante la elevación de la nupcialidad y de los nacimientos una vez superada la crisis -un típico fenómeno de regulación homeostática tras el shock de la mortalidad (Dupâquier, 1972)- es enorme la información disponible, estadística y gráfica ${ }^{5}$. Sería muy prolijo y tedioso enumerar una relación siquiera aproximada de episodios de graves crisis de mortalidad de cualquier naturaleza predominante, donde se repite esa secuencia temporal de caída de la nupcialidad y las concepciones, coincidente con la elevación de las defunciones, y seguida de una recuperación de los matrimonios y los nacimientos. No se trata de un fenómeno peculiar de alguna de las grandes crisis de mortalidad o de las mejor conocidas epidemias del pasado, sino de una constante reacción de los componentes demográficos del movimiento natural con motivo del alza brusca de la morbilidad y la mortalidad $^{6}$

En cuanto a los movimientos migratorios provocados por la epidemia, hay que recordar que la primera reacción, incluso ya ante el temor a su inminencia, era la huida; en realidad, este era el único remedio que los médicos se arriesgaban a recomendar ante los peligros de la peste (Bennassar, 1969, pp. $23-24,52)^{7}$. Sin embargo, aun siendo muy conocidos los relatos

\footnotetext{
${ }^{4}$ Un comentario crítico a esta teoría se desarrolla en Pérez Moreda (2010) y en Pérez Moreda y Collantes (2013). Véase también Livi Bacci (1978b).

${ }^{5}$ Muchos célibes recomponían su compromiso nupcial aplazado por la epidemia o adelantaban la fecha y edad de ingreso en el estado matrimonial estimulados por la herencia de progenitores desaparecidos durante la crisis.

${ }^{6}$ Observable, por ejemplo, tras la gripe española de 1918-1920 (Maluquer, 2020), igual que en tantas otras epidemias y grandes mortandades del pasado.

${ }^{7}$ La recomendación galénica, difundida por Marsilio Ficino - «Cito, longe fugeas (et) tarde redeas»-, se repitió literalmente en España y en otros países, y se popularizó aquí por medio del proverbio de las tres eles : «Huir
}

de huida masiva de la población de la ciudad apestada, y a pesar de la vigilancia y barreras impuestas para impedirla y para garantizar su aislamiento, no era menor el flujo de entradas y salidas de comerciantes, funcionarios públicos y, sobre todo, de gentes del campo y población marginal que acudía, regresaba o intentaba hacerlo burlando los controles o una vez que su rigor se iba relajando (Arráez Tolosa, 2018; MacKay, 2019). Se deben tener en cuenta, por tanto, los movimientos migratorios en ambas direcciones, trasvases de población entre la ciudad sitiada y las zonas rurales: de salida en el primer caso, en busca del refugio de la campiña; y de inmigración a las ciudades en busca de mantenimientos, medios de subsistencia y trabajo por parte de la población de las localidades rurales próximas. Esto último sucedía sobre todo cuando las epidemias castigaban solo o preferentemente a las localidades urbanas (Del Panta, 1980, pp. 98-99; Alfani, 2010a, pp. 172-173; 2020; Alfani y Murphy, 2017; Alfani y Percoco, 2019). A nivel general, en el macroespacio regional o de conjuntos territoriales amplios, los movimientos migratorios reconfiguraron la distribución geográfica del poblamiento, tendiendo a compensar las pérdidas poblacionales de ciudades y núcleos mayores más afectados por la epidemia, lo que explicaría la rápida recuperación demográfica de muchas localidades, aun a costa de la aparición de vacíos o despoblados en el mundo rural ${ }^{8}$.

La mortalidad epidémica puede provocar cambios en la composición interna de la población, más o menos frecuentes e importantes según afecten a la estructura por edades -los de mayores implicaciones económicas-, por sexo o por el estado civil de los habitantes. Respecto a este último cambio en la distribución de la población por estado civil, cabe advertir que se producirá -más allá del aumento temporal de la soltería a causa del retraso y declive de la nupcialidad- si la sobremortalidad epidémica incide de forma especial sobre personas casadas, siempre que no se trate de ambos cónyuges de una misma unidad matrimonial, creciendo así el número y la proporción de viudos/as en el conjunto de la población y de los hogares existentes ${ }^{9}$. En cuanto a la mortalidad diferencial por sexo, no parece ser una característica frecuente en las epidemias históricas, al menos no fácilmente demostrable a través de las fuentes disponibles ${ }^{10}$.

Otros efectos demográficos de la mortalidad epidémica, de consecuencias económicas y sociales también importantes, pueden observarse en la distribución de la población por fa-

de la pestilencia con tres eles es prudencia: luego, lexos y luengo tiempo» (Carreras Panchón, 1976, p. 92).

${ }^{8}$ Véase, por ejemplo, Iradiel (1917, pp. 341-342) respecto al espacio mediterráneo en el periodo bajomedieval y en concreto en el caso valenciano.

${ }^{9}$ La proporción de viudos y viudas, o más concretamente, la de segundas y posteriores nupcias -que contribuían a reducir aquella-, se ha utilizado como un indicador de las tendencias de la mortalidad adulta y general (Nadal, 1984, p. 97; Pérez Moreda, 1986, p. 14).

${ }^{10}$ A pesar de la sobremortalidad femenina que quiso ver Bennassar (1969, pp. 50 y 70) en la peste castellana de 1596-1602, con el ejemplo de solo cuatro localidades. Otros autores, sin embargo, han creído descubrir una mayor incidencia diferencial sobre el sexo masculino en las epidemias de 1603 y 1625 en algunas parroquias londinenses (Hollingsworth, 1971), aunque en otras localidades rurales, también inglesas, algo posteriores como las de Colyton en 1645-1646, o Eyam en 1665-1666, no se puede probar lo mismo al ser afectados ambos sexos por igual o al mostrar el total de las defunciones una sex ratio idéntica a la del periodo anterior a la crisis (Schofield, 1977, p. 109). 
milias u hogares; concretamente el número y tamaño medio de estos últimos tendrían que verse reducidos a consecuencia de la sobremortalidad epidémica ${ }^{11}$. Junto al descenso del número de padres y otros miembros de mayor edad de la familia, también se acusaría el de descendientes actuales y potenciales -estos últimos, en el caso de familias de descendencia incompleta, tras la muerte de uno de los cónyuges en edad aún fértil de la madre. Es decir, la epidemia reduce el número de herederos directos por familia, aunque aumente el volumen agregado de huérfanos sobrevivientes. En poblaciones de predominio de la familia nuclear y aun en sistemas de sucesión igualitaria ello afectará a la transmisión intergeneracional del patrimonio familiar, abriendo la posibilidad de ascenso social para algunos jóvenes adultos; pero lanzará a muchos otros huérfanos a la emigración y a un destino incierto en su horizonte social y económico. Todo lo cual daría lugar a importantes variaciones en los índices de desigualdad de la riqueza.

Los cambios que la epidemia suele introducir en el movimiento y en la estructura de la población dan origen a otra serie de consecuencias económicas, a veces solo temporales y por ello difíciles de medir y de importancia discutible. El retroceso de la fecundidad, junto con el ascenso de la mortalidad, contribuye a reducir el tamaño de la población y frenar el crecimiento demográfico, lo que en algunos casos podría ser una típica solución malthusiana a un desequilibrio previo entre población y recursos. Pero dado que estas alteraciones de los componentes del movimiento natural registraban tendencias compensatorias una vez concluida la crisis epidémica, el crecimiento de la población se reanudaba pronto, aun sin recibir el aporte migratorio. Solo tras una serie de oleadas de grave sobremortalidad, consecutivas, generales y suficientemente próximas entre sí como para anular las ganancias demográficas acumuladas desde la epidemia anterior, la población afectada registrará una tendencia descendente, que si a corto plazo puede aliviar problemas ecológicos o la presión sobre los recursos disponibles, a la larga no hará sino contraer la demanda agregada, reduciendo la inversión total y el crecimiento económico ${ }^{12}$. Por otra parte, el tiempo de recuperación (T) de los efectivos previos de una población sometida a una crisis aislada de mortalidad -a una epidemia, por ejemploguarda, lógicamente, una relación positiva con la intensidad de la misma, medida por la tasa bruta de mortalidad (M) que alcance, y negativa con el saldo vegetativo medio $(n-m)$ durante el periodo posterior al shock epidémico, y puede ser fácilmente calculado en años como $\mathrm{T}=\ln (1-\mathrm{M}) /(m-n)^{13}$.

\footnotetext{
${ }^{11}$ Livi Bacci (1978a, pp.18-20) calculó las consecuencias de una mortalidad de crisis sobre el número y tamaño de las familias. Si la sobremortalidad fuera, por ejemplo, del 30\%, el tamaño medio pasaría de 3 a 2.3, de 4 a 3 , de 5 a 3.6 y de 6 a 4.3 miembros por unidad doméstica, y desaparecerían respectivamente un 10 , un 5 , un 3 y el $1 \%$ de los hogares.

${ }^{12}$ Un sencillo cálculo matemático basado en datos de las pestes que sufre la Toscana entre 1340 y 1450 (un nuevo brote de la epidemia cada nueve años por término medio, con una mortalidad que en cada caso sextuplica la de un año normal, suponiendo invariable la natalidad en el año de la crisis, que se elevaría hasta 10 puntos por 1000 en el intervalo de recuperación postepidémica) arroja una pérdida neta acumulada del $43 \%$ de la población inicial, muy próxima al declive real que se admite para la población europea en ese periodo (Livi Bacci, 1998, pp. 86-87).

${ }^{13}$ Bouckaert (1995, p. 218), donde $n$ y $m$ son respectivamente las tasas brutas de natalidad y mortalidad en el periodo de recuperación postcrisis. El cálculo se aplica en el supuesto de población cerrada, donde el posible saldo migratorio no se aleja significativamente de cero.
}

Sin duda, las más importantes consecuencias económicas del shock epidémico tienen que ver con su incidencia diferencial en distintos grupos de edad de la población. Como ocurre tras cambios más lentos de envejecimiento de la pirámide demográfica y/o aumento del índice de dependencia ${ }^{14}$, la epidemia suele ocasionar, cuando afecta especialmente a los grupos de edades activas (las prime ages en términos económicos), cambios importantes en la oferta de trabajo -con posible elevación de los costes salariales-, en la demanda de bienes de consumo o de capital de acuerdo con las preferencias de los consumidores según su grupo de edad (por lo tanto, en los precios relativos de productos y factores, incluido el coste del capital -el tipo de interés-), en el ahorro y el consumo agregado y en la transferencia intergeneracional de recursos y riqueza (Banco de España, 2018, pp. 225-272; Delivorias y Scholz, 2020; Jordà, Singh y Taylor, 2020; Galesi, Nuño y Thomas, 2017) $)^{15}$.

Hay que tener en cuenta, por otra parte, que la importancia de las consecuencias demográfico-económicas de una enfermedad infecciosa depende en última instancia de la morbilidad y mortalidad que provoque, que a su vez dependen de su letalidad, del grado de infectividad del contagio y de la duración de la epidemia ${ }^{16}$. Toda enfermedad transmisible genera externalidades negativas, altos costes económicos de dos tipos: los directamente dependientes de la morbilidad y la mortalidad y, posiblemente anteriores a ellos y aún más elevados, los costes preventivos. Los primeros son gastos en tratamientos médicos y hospitalización, los costes del absentismo laboral por enfermedad y los derivados de la reducción permanente -difícilmente recuperable a corto y medio plazo- de fuerza de trabajo a causa de la mortalidad de la crisis ${ }^{17}$. Pero la misma prevención, ante el riesgo y el temor al contagio, origina también costes económicos graves de tipo profiláctico, anteriores a la difusión del brote epidémico y que suelen ser proporcionales a las medidas adoptadas para evitarla: sobre todo medidas de confinamiento en sus variantes clásicas de la cuarentena o en su moderna versión del lockdown ajustado a la prevalencia, intensidad y duración previsibles de la enfermedad. Desde hace tiempo, y no solo a raíz de la pandemia actual,

\footnotetext{
14 Que anula las ventajas derivadas del llamado "dividendo demográfico» (Bloom, 2020). Véase Lee et al. (1995) sobre los cambios en la distribución de la población por edad y sus implicaciones económicas en tiempos actuales.

${ }^{15}$ Aunque el análisis de los determinantes de la caída del tipo de interés se deduzca expresamente de «los cambios demográficos experimentados en muchas economías avanzadas» (Galesi, Nuño y Thomas, 2017, pp. 3-5). Naturalmente, muchas de estas consecuencias macroeconómicas cumplen las predicciones de la teoría de Modigliani del ciclo vital del consumo y el ahorro (Modigliani, 1949, 1966).

${ }^{16}$ Se puede admitir, de acuerdo con las simulaciones llevadas a cabo a través de diversos modelos epidemiológicos y económicos, que las consecuencias económicas de una pandemia dependen más de la infectividad o velocidad de transmisión del contagio (el «número básico de reproducción», $\mathrm{R}_{\mathrm{o}}$ ), que de su letalidad: el impacto económico global es mayor en el caso de una infección de transmisión rápida y baja virulencia -aunque arroje un menor número de víctimas mortales- que en el caso contrario de una combinación de alta letalidad y baja infectividad (Verikios et al., 2011, p. 8).

17 Estos costes recaen sobre los individuos, las compañías aseguradoras $\mathrm{y} / \mathrm{o}$ los sistemas de seguridad social, con un gran impacto agregado sobre el conjunto de la economía. Y solo una especial incidencia de la mortalidad sobre las edades superiores reduce los costes individuales (gastos médicos y asistenciales) y los gastos sociales (pensiones, p. ej.) (Bell y Lewis, 2004).
} 
ha quedado establecido que estos costes preventivos se manifiestan principalmente en tres tipos de comportamientos, de origen espontáneo o promovidos por los poderes públicos: a) reducción del gasto de los hogares en bienes de consumo diferible, en transportes y en ocio y turismo; b) caída del producto final y aumento del absentismo laboral por cierre temporal de centros de producción industrial o de servicios, y c) aumento del absentismo laboral en familias con hijos en edad escolar por cierre de los centros educativos ${ }^{18}$.

En cualquier caso, los costes económicos de la epidemia serán de un tipo u otro dependiendo de cuál sea el grupo o los grupos de edades más afectados: si se trata de los adultos jóvenes en edades laborales, una inmediata reducción de la oferta de trabajo, manteniéndose estable el stock de capital fijo, presionará los salarios al alza si la tasa previa de desempleo no es elevada; se reducirá también la fecundidad, y por ello la fuerza de trabajo futura, y se resentirá la educación y la formación de capital humano de los menores. Si la morbilidad y la mortalidad castiga preferentemente a estos últimos o a las edades superiores, los efectos sobre el mercado laboral serán, en principio, nulos o solo parciales, pero en ambos casos se desvían recursos familiares a la atención de los dependientes enfermos, con repercusión negativa en el PIB y, a largo plazo, también en el volumen y calidad de la oferta agregada de trabajo (Bell y Lewis, 2004).

\section{Consideraciones finales}

Apenas nada de lo expuesto anteriormente desciende a los detalles de otras posibles consecuencias económicas derivadas de la epidemia y de los cambios demográficos que directamente provoca. Algunas han quedado semiocultas o solo ligeramente aludidas, pues no se manifiestan en el momento de la crisis, sino que las padecen los individuos y las sociedades más tarde, a muy largo plazo. Son por ejemplo los efectos de onda, o de generaciones vacías, que cíclicamente afectarán al mercado de trabajo, y posiblemente también al mercado matrimonial y a la fecundidad, durante un largo periodo tras el episodio epidémico (Easterlin, 1968, 1978) ${ }^{19}$. Otros efectos de la epidemia serán, como ya se ha señalado, los daños que afectan seriamente a la educación de los menores, directamente por el cese temporal de la escolarización o indirectamente por la morbilidad y mortalidad de padres y abuelos ${ }^{20}$. La crisis en

\footnotetext{
${ }^{18}$ Verikios et al. (2011, pp. 12-13) cuantifican en sus modelos solo algunos de estos costes preventivos de la epidemia e introducen otros como los gastos médicos y hospitalarios. Véase también Eichenbaum et al. (2020). Todas esas actuaciones del público suelen traer aparejadas otras importantes consecuencias económicas negativas, como la caída de la recaudación fiscal y de los ingresos públicos en general. Son estas últimas, junto con los gastos sociales que solían afrontar los municipios con motivo de la enfermedad masiva, casi las únicas consecuencias económicas, medidas en términos monetarios, de las que nos ha llegado información relativa a las epidemias de épocas antiguas (Cipolla, 1976, pp. 57 y ss.; 1981, pp. 66-76; Del Panta, 1980, pp. 97-98).

${ }^{19}$ Es clásico entre nosotros el artículo de Nadal (1990) donde se muestra la sucesión de al menos tres periodos sucesivos de generaciones diezmadas en la Cataluña del siglo XIX que acusan los efectos de la sobremortalidad de 1793-1812.

${ }^{20}$ En relación con los costes en productividad a largo plazo que impondrá la actual crisis de la COVID-19, Rogoff (2020) ha pronosticado que una vez superada la pandemia una generación de niños, sobre todo los de familias de menores ingresos, habrá perdido todo un año de escolarización.
}

general, y sobre todo el incremento de orfandad que ocasiona, tiene efectos muy negativos sobre el stock de capital humano de las generaciones infantiles y juveniles que la sufren (Bell y Davis, 2004).

Cabe añadir, en este contexto, otro tipo de efectos negativos sobre la población de las primeras edades infantiles a causa de la morbilidad y la restricción alimentaria que padecen esas cohortes de la crisis (los que nacen o dan sus primeros pasos en medio de todas las penalidades y estrecheces de la epidemia o del periodo postepidémico), y cuyos efectos deletéreos, sanitarios y económicos, se visualizan a largo plazo. Se trata de los daños en la salud -incluidas las habilidades cognitivas-, cuyos efectos repercuten y son observables primeramente en el rendimiento escolar, y más tarde en el capital humano y la futura calidad y remuneración de la mano de obra de esas generaciones a su llegada al mercado laboral; son los efectos previstos en la "hipótesis Barker», suficientemente comprobados en la literatura médica y demográfica, y verificables también en las secuelas de algunas de las grandes epidemias y pandemias históricas ${ }^{21}$.

Otra relevante característica de los episodios epidémicos tiene que ver con la incidencia diferencial de la morbilidad y mortalidad de la crisis sobre los grupos, clases y sectores sociales de diferente estatus y nivel de ingresos. Cuestión importante y compleja, que cuenta con un enorme volumen de información procedente de las fuentes contemporáneas y ha sido tratada prácticamente en todas las monografías sobre las epidemias históricas, que invariablemente, hasta tiempos muy recientes, han dado por buena la relación directa entre sobremortalidad y pobreza que apenas sin excepción denunciaron durante siglos los médicos y tratadistas de la peste o los cronistas de las otras importantes enfermedades transmisibles de siglos pasados (Defoe, 1969 [1722], pp. 102, 107, 245-246; Baehrel, 1952; Biraben, 1971; Bennassar, 1969, pp. 53-54; Carreras, 1976, pp. 123-124; Evans, 1987, pp. 403-469; 1988; Fernández Sanz, 1990, pp. 299-301; Wade, 2020). A pesar de que también desde los tiempos posteriores a la peste negra abundan las referencias a su mortalidad como niveladora de las diferencias sociales ${ }^{22}$, una gran mayoría de historiadores han admitido que las epidemias, como las grandes mortandades en general, afectaron mucho más a los sectores de la población de menos recursos o situados en el límite de la subsistencia, cuyas defensas inmunitarias estaban sumamente debilitadas tras años de carestía.

El tema, pues, se complica con la introducción del factor alimentario como uno de los desencadenantes decisivos de la epidemia, y se ha visto sujeto a la discusión científica en tiempos recientes y actuales, por lo que merecería por sí solo una reflexión monográfica sobre la cual no podemos ahora exten-

\footnotetext{
${ }^{21}$ Acerca de los efectos sobre la salud adulta del deterioro nutricional padecido en el periodo fetal o postnatal existe abundante literatura médica, de la que solo citaremos al propio Barker (1992, 1998; Barker and Osmond, 1986); y también muchas comprobaciones históricas, buena parte de ellas relativas a los efectos a largo plazo de la gripe española de 1918-1920 sobre el desarrollo físico e intelectual de los supervivientes de las cohortes de la epidemia (Almond, 2006; Parman, 2015; Vollmer and Wójcik, 2017; Saavedra, 2017; Palloni et al., 2020; Spinney, 2020, pp. 225 226).

${ }^{22}$ De lo que es ejemplo, entre otros muchos, la famosa reflexión de Jorge Manrique: "[...] que a papas y emperadores/y prelados/así los trata la muerte/como a los pobres pastores/de ganados" (Coplas a la muerte del maestre Don Rodrigo Manrique, c. 1470, estrofa 14).
} 
dernos. Solo habrá que recordar, no obstante, que la relación entre desnutrición -agravada por la carestía- e infección no es uniforme en todas las enfermedades infecciosas, siendo mucho más evidente en algunas como la tuberculosis, el cólera, el sarampión o el tifus que en otras como la peste o la viruela (Lunn, 1991). Y que, por otra parte, la secuencia de los hechos históricos, que por lo general se ha presentado de una forma lineal e invariable, no encaja con la realidad de muchas de las grandes epidemias del pasado, en las que la carestía y el hambre no anteceden en muchos casos a la mortalidad, ni siquiera son simultáneos a la crisis, sino posteriores, por lo que no parecen ser su causa o uno de sus factores, sino más bien su consecuencia (Brumont, 1988; Lázaro Ruiz, 1994, pp. 54-57; Pérez Moreda, 2010; Pérez Moreda y Collantes, 2013, pp. 3334; Defoe, 1969 [1722], p. 115). La disminución de la oferta por la morbilidad y mortalidad de la crisis, junto a las medidas de confinamiento impuestas por las autoridades en su lucha contra la difusión de la enfermedad, que distorsionaban o impedían el comercio a gran distancia (cuarentenas, lazaretos portuarios) y la distribución de subsistencias a las localidades epidemiadas (cordones sanitarios, cierre de las puertas de la ciudad), conducían a la carestía y el hambre. Sucede hoy otro tanto a causa de la pandemia de COVID-19, que está generando o a punto de agravar serios problemas de hambruna masiva, sobre todo en los países en vías de desarrollo, como denuncian las organizaciones internacionales y destacados analistas económicos (Ravallion, 2020; Reinhart y Subbaraman, 2020)23.

Son estas algunas de las otras consecuencias importantes que puede provocar una pandemia como la actual, o como tantas otras que la han precedido. Muchas de ellas, así como las causas y otras características de estas graves crisis -epidemiológicas y sanitarias, sociales y culturales, económicas y ambientales- se repiten frecuentemente porque son comunes a todas ellas. No obstante, un verdadero escrutinio de los episodios epidémicos requiere una detenida labor histórica que descienda y explique las particularidades de cada caso. La economía, y en concreto la historia económica, como reconocía Rosenberg, tienen esa misión, sin la cual no podremos llegar a reconstruir esa "etnografía" y esa "ecología» de las epidemias (Rosenberg, 1992, pp. 303-304), indispensables para el verdadero conocimiento de las del pasado, las del presente y las que muy probablemente seguirán llegando en el futuro.

\section{Bibliografía}

Alfani, G., 2010a. Il Grand Tour dei Cavalieri dell'Apocalisse. Economia, Popolazione e Calamità nell' Italia del Cinquecento. Marsilio Editori, Venezia.

Alfani, G., 2010b. Pestilenze e 'crisi di sistema' in Italia tra XVI e XVII secolo. Perturbazioni di breve periodo o cause di declino economico?. En: Cavaciocchi, S. (ed.). Le interazione fra economia e ambiente biologico nell'Europa preindustriale sec. XIII-XVIII. Firenze University Press, Firenze, pp. 219-244. Alfani, G., 2020. Pandemics and asymmetric shocks: Lessons from the history of plagues. [disponible en: https://voxeu.org/debates/economics-time-covid-19]. Alfani, G. y Murphy T. E., 2017. Plague and Lethal Epidemics in the Pre-Industrial World. Journal of Economic History, 77 (1), 314-343.

\footnotetext{
${ }^{23}$ Los medios -Ibercampus de 10 de mayo, El Confidencial de 1 de junio (Torrico, 2020) - difundían la preocupación de la FAO, UNICEF o la OMS por el problema de la alimentación mundial. Ya antes The Lancet alertaba sobre las probables "lockdown victims» y el grave riesgo de crisis alimentaria que se cernía sobre amplios territorios, sobre todo del África Subsahariana, según pronósticos del Banco Mundial y la Organización Mundial del Comercio (Horton, 2020).
}

Alfani, G. y Percoco, M., 2019. Plague and long-term development: the lasting effects of the 1629-30 epidemic in the Italian cities. Economic History Review, 72 (4), 1175-1201.

Almond, D., 2006. Is the 1918 Influenza Pandemic over? Long-term Efects of in utero Influenza Exposure in the post-1940 U.S. Population. Journal of Political Economy, 114 (4), 672-712.

American Anthropologist Association, 2020. Disease and Pandemics, April 17 (disponible en: http://www.americananthropologist.org/2020/04/17/disease-and-pandemics/).

Appleby, A. B., 1973. Disease or famine? Mortality in Cumberland and Westmorland, 1580-1640. Economic History Review, 26 (3), 403-432.

Appleby, A. B., 1977. Famine, mortality, and epidemic disease: a comment. Economic History Review, 30 (3), 508-512.

Asociación Española de Geografía, 2020. AGE y Pandemia. Aportaciones (disponible en: https://www.age-geografia.es/site/reflexiones-sobre-la-crisis-actual/).

Asociación Española de Historia Económica (AEHE), 2020. La historia económica en los medios: COVID-19 (disponible en: https://www.aehe.es/articulos-en-prensa-de-los-socios-aehe-2-2-2-2-2/)

Arráez Tolosa, A., 2018. El paso de la epidemia de la peste atlántica de 1596-1602 por Almansa. Al-Basit. Revista de Estudios Albacetenses, XLII, 63, 175-214.

Baehrel, R., 1952. La haine de classe en temps d'épidémie. Annales, E. S. C., 7 (3), 351-360.

Banco de España., 2018. Informe anual 2018. Banco de España, Madrid.

Bardet, J. P., Bourdelais, P., Guillaume, P., et al. (dirs.), 1988. Peurs et terreurs face à la contagion. Fayard, Paris.

Barker, D. J. P., 1992. The Fetal and Infant Origins of Adult Disease. BMJ Books, London.

Barker, D. J. P., 1998. Mothers, babies and disease in later life. Churchill Livingstone, Edinburgh.

Barker, D. J. P., y Osmond, C., 1986. Infant mortality, child nutrition, and ischaemic heart disease in England and Wales. The Lancet, 1, 1077-1081.

Bell, C. y Lewis M., 2004. The Economic Implications of Epidemics Old and New. World Economics, 5 (4, October), repr. en Center for Global Development. Working Paper, 54.

Bennassar, B., 1969. Recherches sur les grandes épidémies dans le Nord de l'Espagne à la fin du XVIe siècle. S. E. V. P. E. N., Paris.

Biraben, J. N., 1971. Les pauvres et la peste. Médecine et maladies infectieuses, I (7-8), 313-320.

Biraben, J. N., 1975. Les hommes et la peste en France et dans les pays européens et méditerranéens. I: La peste dans l'histoire. Mouton, Paris-La Haye.

Bloom, D. E., 2020. Demographics can be a potent driver of the pace and process of economic development. Finance and Development, 57 (1), 6-9.

Bouckaert, A., 1995. Crisis mortality: extinction and near-extinction of human populations. In: Ruzicka, L., Wunsch, G. y Kane, P (eds.). Differential mortality. Methodological Issues and Biosocial Factors. Oxford University Press, New York, pp. 217-230.

Brumont, F., 1988. Le pain et la peste: épidémie et subsistances en Vieille-Castille à la fin du XVIe siècle. Annales de Démographie Historique, 207-220.

Campos, R., Perdiguero-Gil, E. y Bueno, E. (eds.), 2020. Cuarenta historias para una cuarentena. Reflexiones históricas sobre epidemias y salud global. Sociedad Española de Historia de la Medicina, Madrid.

Carreras Panchón, A., 1976. La peste y los médicos en la España del Renacimiento. Universidad de Salamanca, Salamanca.

Cássia da Silva, R., Goodwin, J. W. Jr., y Saraiva, L. F. (orgs.), 2020. Na saúde e na doença. História, crises e epidemias. Reflexões da História Econômica na época da Covid-19. Hucitec Editora, São Paulo.

Cipolla, C. M., 1976. Public Health and the Medical Profession in the Renaissance. Cambridge University Press, London.

Cipolla, C. M., 1981. Fighting the Plague in Seventeenth-Century Italy. The University of Wisconsin Press, Madison-Wisconsin.

Consejo Superior de Investigaciones Científicas (CSIC), 2020a. Salud Global-Global Health COVID 19. Principales novedades internacionales sobre SARS-Cov-2. Newsletters PTI (11-mayo/30-julio, 2020) (disponible en: https://pti-saludglobal-covid19.corp.csic.es/newsletter/).

Consejo Superior de Investigaciones Científicas (CSIC) 2020b. Una visión global de la pandemia COVID-19: qué sabemos y qué estamos investigando desde el CSIC (disponible en: https://digital.csic.es/handle/10261/218312).

Defoe, D. 1722. A Journal of the Plague Year: being Observations or Memorials of the most Remarkable Occurrences, as well publick as private, which happened in London during the last Great Visitation in 1665 (trad. española, 1969. Diario del año de la peste. Seix Barral, Barcelona).

Del Panta, L., 1980. Le epidemie nella storia demografica italiana (secoli XIV-XIX) Loescher Editore, Milano.

Delivorias, A. y Scholz, N., 2020. Economic impact of epidemics and pandemics. European Parliamentary Research Service, PE 646.195. 
Dupâquier, J., 1972. De l'animal à l'homme: le mécanisme autorégulateur des populations traditionnelles. Revue de l'Institut de Sociologie (Bruxelles), 45 (2), 177-211.

Easterlin, R. A., 1968. Population, Labor Force and Long Swings in Economic Growth: the American Experience. Columbia University Press, New York

Easterlin, R. A., 1978. What will 1984 will be like? Socioeconomic implications of recent twists in age structure. Demography, 15 (4), 397-432.

Economic History Society, 2020a. The Long View on Epidemics, Disease and Public Health: Research from Economic History, Part A, March 26 (disponible en: https://ehsthelongrun.net/2020/03/26/the-long-view-on-epidemicsdisease-and-public-health-research-from-economic-history-part-a/).

Economic History Society, 2020b. The Long View on Epidemics, Disease and Public Health: Research from Economic History, Part B, March 31 (disponible en: https://ehsthelongrun.net/2020/03/31/the-long-view-on-epidemicsdisease-and-public-health-research-from-economic-history-part-b/).

Economic History Society, 2020c. The Long View on Epidemics, Disease and Public Health: Research from Economic History, Part C, April 18 (disponible en: https://ehsthelongrun.net/2020/04/18/the-long-view-on-epidemicsdisease-and-public-health-research-from-economic-history-part-c/).

Eichenbaum, M. S., Rebelo, S. y Trabandt, M., 2020. The Macroeconomics of Epidemics. NBER Working Paper, 26882

Evans, R. J., 1987. Death in Hamburg. Society and Politics in the Cholera Years, 1830-1910. Oxford University Press, Oxford

Evans, R. J., 1988. Épidémies et révolutions. Le choléra dans l'Europe du XIX siècle. En: Bardet J. P. et al. (dirs.). Peurs et terreurs face à la contagion. Fayard, Paris, pp. 107-135.

Fernández Sanz, J. J., 1990. 1885: el año de la vacunación Ferrán. Trasfondo político, médico, sociodemográfico y económico de una epidemia. Fundación Ramón Areces, Madrid.

Fundación de Ciencias de la Salud (2020). Desde la memoria: historia, medicina y ciencia en tiempos de... (disponible en: https://www.fcs.es/publicaciones/ publicaciones).

Galesi, A., Nuño, G. y Thomas C., 2017. El tipo de interés natural: concepto, determinantes e implicaciones para la política monetaria. Banco de España. Boletín Económico 1/2017: Artículos analíticos.

Goubert, P., 1965. La mortalité en France sous l'Ancien Régime. Problèmes et hypothèses. Problèmes de mortalitè. Actes du Colloque International de Démographie Historique (Liège, avril 1963). Paris, pp. 79-92.

Hollingsworth, M. F. and T. H., 1971. Plague mortality rates by age and sex in the parish of St. Botolph's without Bishopsgate, London, 1603. Population Studies, 25 (1), 131-146.

Horton, R., 2020. A call for a post-pandemic health strategy. The Lancet, 395 (April 18), 1242.

Institut National d'Études Démographiques (INED) 2020. Crise sanitaire et confinement: l'apport de la démographie et des sciences de la population (disponible en: https://www.ined.fr/fr/ressources-methodes/etat-de-la-recherche/covid-19/).

Iradiel, P., 2017. Consecuencias económicas y demográficas de las epidemias del siglo XV en la Corona de Aragón. En: Iradiel, P. El Mediterráneo medieval y Valencia. Economía, sociedad, historia. Universitat de València, Valencia, pp. 323-345.

Jordà, O., Singh, S. R. y Taylor A. M., 2020. Longer-Run Economic Consequences of Pandemics. Federal Reserve Bank of San Francisco, Working Paper, 2020-09.

Lázaro Ruiz, M., 1994. La población de la ciudad de Logroño durante el Antiguo Régimen (1500-1833). Gobierno de la Rioja, Instituto de Estudios Riojanos, Logroño.

Lee, R. D., Arthur, W. B., y Rodgers, G. (eds.) 1995. Economics of Changing Age Distributions in Developed Countries. Oxford University Press, New York.

Livi Bacci, M., 1978a. La société italienne devant les crises de mortalité. Dipartimento Statistico, Università degli Studi di Firenze, Firenze.

Livi Bacci, M., 1978b. Les répercussions d'une crise de mortalité sur la fécondité: une vérification empirique. Annales de Démographie Historique, 197-207.

Livi Bacci, M., 1998. Historia de la población europea. Crítica, Barcelona.

Livi Bacci, M. (ed.) 2020. L'ospite inatesso. Neodemos e il COVID-19. Associazione Neodemos, Firenze.

Lunn, P. G., 1991. Nutrition, immunity and infection. En: Schofield et al. (eds.). The decline of mortality in Europe. Clarendon Press, Oxford, pp. 131-145.

MacKay, R., 2019. Life in a time of pestilence. The Great Castilian Plague of 15961601. Cambridge University Press, Cambridge.

Maluquer de Motes, J., 2020. La "madre de todas las pandemias». Conversación sobre la Historia (disponible en: https://conversacionsobrehistoria. info/2020/08/02/la-madre-de-todas-las-pandemias-impacto-demografico-de-la-gripe-de-1918-1920/).

Meuvret, J., 1965a. Réflexions d'un historien sur les crises démographiques aigües avant le XVIIIe siècle. Problèmes de mortalitè. Actes du Colloque International de Démographie Historique (Liège, avril 1963). Paris, pp. 93-97.

Meuvret, J., 1965b. Demographic crises in France from the Sixteenth to the Eighteenth Century. En: Glass, D. V. y Eversley, D. E. C. (eds.). Population in History. Essays in Historical Demography. Edward Arnold, London, pp. 507522 .

Modigliani, F., 1949. Fluctuations in the saving-income ratio: a problem in economic forecasting. Studies in Income and Wealth. Vol. 11, NBER, New York, pp. 371-443.

Modigliani, F., 1966. The Life Cycle Hypothesis of Saving, the Demand for Wealth and the Supply of Capital. Social Research. 33 (2), 160-217.

Nadal, J., 1984. La población española (Siglos XVI a XX). Ariel, Barcelona.

Nadal, J., 1990. Las grandes crisis de mortalidad de los años 1793-1812: los efectos a largo plazo en la población catalana. Boletín de la Asociación de Demografía Histórica, 8 (2), 37-49.

Palloni, A. et al., 2020. Impacts of the 1918 flu on survivors' nutritional status: a double quasi-natural experiment. Working Paper (disponible en: https://doi. org/10.1101/2020.04.23.057638).

Parman, J., 2015. Childhood Health and Sibling Outcomes: Nurture reinforcing Nature during the 1918 Influenza Pandemic. Explorations in Economic History, 58, 22-43.

Pérez Moreda, V., 1980. Las crisis de mortalidad en la España interior (siglos XVI-XIX). Siglo XXI, Madrid.

Pérez Moreda, V., 1986. Matrimonio y familia. Algunas consideraciones sobre el modelo matrimonial español en la Edad Moderna. Boletín de la Asociación de Demografía Histórica, 4 (1), 3-51.

Pérez Moreda, V., 2010. Una nueva interpretación de las relaciones entre mortalidad y economía: pruebas históricas en contra del modelo de las «crisis de subsistencias». En: Cavaciocchi, S. (ed.). Le interazione fra economia e ambiente biologico nell'Europa preindustriale sec. XIII-XVIII. Firenze University Press, Florencia, pp. 181-218.

Pérez Moreda, V. y Collantes, F., 2013. Crisis demográficas del pasado y problemas demográficos del presente. En: Comín, F. y Hernández, M. (eds.). Crisis económicas en España, 1300-2012. Lecciones de la historia. Alianza Editorial, Madrid, pp. 27-54.

Ravallion, M., 2020. Could Pandemic Lead to Famine? Project Syndicate. April 15.

Reinhart, C. and Subbaraman, R., 2020. Preventing a Covid-19 Food Crisis. Project Syndicat. May 15.

Rogoff, K., 2020. The Uncertainty Pandemic. Project Syndicate. Sept 3.

Rosenberg, Ch. E., 1992. Explaining epidemics, and other studies in the history of medicine. Cambridge University Press, Cambridge.

Saavedra, M., 2017. Early-life Disease Exposure and Occupational Status: The Impact of Yellow Fever during the 19th Century. Explorations in Economic History, 64, 62-81.

Société de Démographie Historique (SDH/ADH), 2020. L’apport de la démographie historique à l'étude des épidémies et à la compréhension des événements actuels, 20, septiembre (disponible en: https://twitter.com/SDH_demohisto/ status/1257676523231985665).

Schofield, R., 1977. An Anatomy of an Epidemic: Colyton, November 1645 to November 1646. En: The Cambridge Group for the History of Population and Social Structure (ed). The Plague Reconsidered. A new look at its origins and effects in 16th and 17th Century England. Local Population Studies Supplement, Hourdsprint-Cambridge, pp. 95-126.

Spinney, L., 202033. El jinete pálido. 1918: la epidemia que cambió el mundo. Crítica, Barcelona

Torrico, E., 2020. El COVID que todo lo puede: «Existe el riesgo de tener un problema de alimentos en 2021». El Confidencial. 1-6-2020.

Verikios, G. et al., 2011. The Global Economic Effects of Pandemic Influenza. Centre of Policy Studies. General Paper, G-224.

Vollmer, S. y Wójcik, J., 2017. The long-term consequences of the global 1918 influenza pandemic: A systematic analysis of 117 IPUMS international census data sets. Courant Research Centre- Georg-August Universität Göttingen, Discussion Papers, 242.

Wade, L., 2020. From Black Death to fatal flu, past pandemics show why people on the margins suffer most. Science. May 14 (disponible en: doi:10.1126/ science.abc7832) 